\title{
Association of Polymorphisms in the Regulatory Region of the Cyclophilin a Gene (PPIA) with Gene Expression and HIV/AIDS Disease Progression
}

\author{
Paradise Madlala, PhD, * Ravesh Singh, PhD, * Ping An, MD, MPH, † Lise Werner, MSc, $\neq$ \\ Koleka Mlisana, MBChB, PhD, $\neq$ Salim S. Abdool Karim, MBChB, PhD, $\neq$ Cheryl A. Winkler, PhD, $\dagger$ \\ and Thumbi Ndung'u, BVM, PhD* $\S$
}

Background: Human cyclophilin A (CypA) encoded by peptidyl prolyl isomerase A gene (PPIA), enhances HIV-1 replication by aiding capsid uncoating. The association of genetic variation in the PPIA regulatory region with susceptibility to HIV-1 infection, disease progression, and gene expression among black South Africans at risk for infection or infected with HIV-1 is unknown.

\begin{abstract}
Methods: We genotyped 539 participants from 2 longitudinal study cohorts of black South Africans at high risk for infection or infected with HIV-1 for PPIA regulatory single nucleotide polymorphisms by polymerase chain reaction-restriction fragment length polymorphism.
\end{abstract}

Results: Minor allele (G) of SNP rs6850 (rs6850 G) significantly associated with higher viral loads (mean 4.85 versus $4.46 \mathrm{log}$ copies/mL, $P=0.0006$ ) and lower $\mathrm{CD}^{+}{ }^{+}$T-cell counts (mean 506

Received for publication October 26, 2015; accepted April 4, 2016.

From the *HIV Pathogenesis Programme, Nelson R Mandela School of Medicine, University of KwaZulu-Natal, Durban, South Africa; †Centre for the AIDS Programme of Research in South Africa (CAPRISA), Nelson R Mandela School of Medicine, University of KwaZulu-Natal, Durban, South Africa; $\uparrow$ Basic Research Laboratory, Center for Cancer Research, National Cancer Institute, Leidos Biomedical Research, Inc., Frederick National Laboratory for Cancer Research, Frederick, MD; and §KwaZulu-Natal Research Institute for Tuberculosis and HIV, Nelson R Mandela School of Medicine, University of KwaZulu-Natal, Durban, South Africa.

Supported by the seventh framework programme (FP7) of the European Commission (THINC, HEALTH-F3-2008-201032). Additional funding was provided by the South African Department of Science and Technology/National Research Foundation Research Chairs Initiative and the Victor Daitz Foundation. Part of the work was funded by the Howard Hughes Medical Institute International Early Career Scientist award to TN. This project has been funded in part with federal funds from the National Cancer Institute, National Institutes of Health, under contract HHSN261200800001E. This Research was supported in part by the Intramural Research Program of the NIH, National Cancer Institute, Center for Cancer Research. The content of this publication does not necessarily reflect the views or policies of the Department of Health and Human Services, nor does mention of trade names, commercial products, or organizations imply endorsement by the US Government.

The data reported here were reported in part at the XIX International AIDS Conference July 22-27, 2012, Washington DC.

The authors have no funding or conflicts of interest to disclose.

Correspondence to: Thumbi Ndung'u, BVM, PhD, HIV Pathogenesis Programme, Doris Duke Medical Research Institute, Nelson R. Mandela School of Medicine, University of KwaZulu-Natal, Level 1, Room 117, Durban 4013, South Africa (e-mail: ndungu@ukzn.ac.za).

Copyright (C) 2016 Wolters Kluwer Health, Inc. All rights reserved. versus 557 cells $/ \mu \mathrm{L}, P=0.0256)$ during the acute phase of infection in the Centre for the AIDS Programme of Research in South Africa (CAPRISA) 002 cohort. Consistently, rs6850 G significantly associated with higher viral loads (mean 4.49 versus $4.01 \mathrm{log}$ copies $/ \mathrm{mL}, P<0.0001$ ) and lower $\mathrm{CD}^{+}$T-cell counts (mean 442 versus 494 cells $/ \mu \mathrm{L}, P=0.0002$ ) during the early chronic phase of infection in the CAPRISA 002 cohort; rs6850 G further associated significantly with rapid $\mathrm{CD}^{+}$T-cell decline in the CAPRISA 002 cohort $(P=0.0481)$ and Sinikithemba chronic infection cohort $(P=$ 0.0156). Interestingly, rs6850 G significantly associated with elevated CypA mRNA levels in HIV-1-positive individuals $(P=0.0061)$.

Conclusions: These data suggest that rs6850 G enhances HIV-1 replication through upregulation of CypA expression following HIV-1 infection. The data support ongoing efforts to develop anti-HIV-1 drugs that block interaction of HIV-1 and cellular proteins.

Key Words: SNP, CypA, PPIA, HIV-1, mRNA expression

(J Acquir Immune Defic Syndr 2016;72:465-473)

\section{INTRODUCTION}

Human cyclophilin A (CypA) is a cellular cofactor for HIV-1 replication. ${ }^{1-3}$ CypA, also known as peptidyl prolyl isomerase A (PPIA), belongs to the cyclophilin family, members of which all possess peptidyl prolyl cis/trans isomerase activity (by convention, the protein is referred to as CypA and the gene as PPIA). Peptidyl prolyl cis/trans isomerases catalyze the cis/trans isomerization of prolyl peptide bonds and are involved in protein folding. ${ }^{4}$ After viral entry into the cytoplasm, CypA is believed to play a role in uncoating of the viral core based on its peptidyl propyl isomerase activity. HIV-1 capsid (CA) directly interacts with the active sites of CypA through a prolyl peptide bond located in a proline-rich loop between the fourth and fifth helix loops and this leads to the incorporation of CypA into the HIV-1 virion capsid. ${ }^{5,6}$

Disruption of the interaction between CypA and HIV-1, either by HIV-1 Gag mutations or by cyclosporine A, an immunosuppressive drug that prevents HIV-1 Gag binding to CypA, leads to attenuation of HIV-1 infectivity. ${ }^{3,7}$ HIV-1 replication was significantly inhibited in CypA-null human $\mathrm{CD}^{+} \mathrm{T}$ cells, in which PPIA was deleted through homologous 
recombination. CypA is therefore an important host factor required for efficient HIV-1 infection. ${ }^{8}$ CypA increases viral infectivity by facilitating proper uncoating. In addition, binding of CypA to capsid protects HIV-1 from an unknown restriction factor in humans. ${ }^{9}$

Although different groups have identified single nucleotide polymorphisms (SNPs) in the regulatory region of PPIA that affect HIV-1 clinical outcomes in different populations, ${ }^{10-12}$ the data are inconsistent and populations studied bear less burden of HIV-1/AIDS compared with the situation in sub-Saharan Africa. ${ }^{13}$ Bleiber et $\mathrm{al}^{11}$ demonstrated an association of the minor allele $(\mathrm{G})$ of SNP rs6850 (referred to as rs6850 G henceforth) with rapid disease progression in a Swiss cohort. Subsequently, An et $\mathrm{al}^{10}$ reported higher frequency of rs6850 G among HIV-1 seroconverters (SC) compared with the high-risk seronegative (SN) group suggesting an association with increased susceptibility to HIV-1 infection. Although there was a nonsignificant association of $\mathrm{rs} 6850 \mathrm{G}$ with CD4 cell depletion in African Americans, no evidence of association was observed in European Americans. In contrary, rs6850 G was significantly increased in high-risk seronegative group of the Amsterdam Cohort Studies (ACS) compared with HIV-1-infected group suggesting that rs6850 $\mathrm{G}$ may be associated with reduced susceptibility to HIV-1 infection in participants of the ACS. ${ }^{12}$ An et $\mathrm{al}^{10}$ found $\mathrm{rs} 8177826 \mathrm{G}$, which is in perfect linkage disequilibrium with SNP rs8177830, to be associated with accelerated progression to AIDS in African Americans. Consistently, Rits et al ${ }^{12}$ demonstrated that $\mathrm{rs} 8177826 \mathrm{G}$ was associated with accelerated progression as assessed by viral load threshold.

The present study was undertaken in an attempt to shed more light on the role of PPIA promoter polymorphisms on HIV-1 pathogenesis in an African population. Two SNPs rs8177830 and rs6850 were selected because previous studies, conducted in American and European cohorts had reported the association of rs 8177830 with rapid disease progression and inconsistent findings were reported regarding the association of rs6850 with susceptibility to HIV-1 acquisition. Specifically, we wanted to address the role of PPIA polymorphisms in a sub-Saharan population in which HIV-1 prevalence is high because there is paucity of data for the role of human genetic variation in HIV/AIDS pathogenesis for this population and because novel therapeutic interventions would be most relevant for this setting. Furthermore, we sought to explore the possible mechanisms by which PPIA polymorphisms may affect HIV-1 pathogenesis. We focused on PPIA expression levels because of previous in vitro data suggesting that CypA levels are largely responsible for differences in HIV replication between CEM-GXR and Jurkat cells. ${ }^{14}$ We thus investigated the association of 2 SNPs, rs8177830 and rs6850, with susceptibility to HIV-1 infection, disease progression, and CypA mRNA levels in a population of black South Africans that has a high burden of HIV-1 infection.

\section{METHODS}

\section{Study Participants}

Participants in this study were from 2 study cohorts of a black South African population in KwaZulu-Natal,
Durban, South Africa. The first cohort was the Centre for the AIDS Programme of Research in South Africa (CAPRISA) 002 cohort. $^{15}$ The CAPRISA 002 cohort is an ongoing observational natural history study of HIV-1 subtype C infection established in Durban, South Africa in 2004. HIV-1-negative women $(n=245)$ at high risk for HIV-1 infection were enrolled into phase I of the study. Participants in this cohort were screened monthly for recent HIV-1 infection by 2 rapid HIV-1 antibody tests, Determine (Abbott Laboratories, Tokyo, Japan) and Capillus (Trinity Biotech, Jamestown, NY). HIV-1 antibody-negative samples were tested for HIV-1 RNA in batches of 10 plasma samples per pool using the Ampliscreen v1.5 assay (Roche Diagnostics, Rotkreuz, Switzerland), which has a detection limit of 10 copies per milliliter. Samples that tested positive in pooled plasma were individually tested by quantitative RNA (Amplicor v2.0, Roche Diagnostics) and HIV enzyme immunoassay (BEP 2000; Dade Behring, Marburg, Germany) to identify the HIV-1-positive sample. $\mathrm{CD}^{+}{ }^{+}$T-cell counts were determined by a 4-parameter FACSCalibur flow cytometer (Becton Dickinson, Franklin Lakes, NJ). Twenty-eight participants seroconverted with acute HIV-1 infection were enrolled into phase II of the CAPRISA 002 study on the basis of a reactive HIV antibody test within 5 months of previously negative results or positive HIV RNA polymerase chain reaction (PCR) in the absence of antibodies. A further 34 acutely HIV-1 subtype C infected participants from other the seroincidence studies of black South Africans from KwaZulu-Natal were also enrolled into phase II if they met the above criteria for inclusion in the acute infection phase, therefore establishing a cohort of 62 HIV seroconverters, all antiretroviral naive. For the purposes of this study, samples from 168 HIV SN participants and 47 participants with recent HIV infection (SC) were available and used for analysis. Date of infection was estimated by taking the midpoint between the last HIV antibody-negative result and the first HIV antibody-positive result or 14 days before the first positive HIV RNA PCR assay result for those identified as antibody negative but HIV RNA positive. Participants in phase II were intensively followed-up by monitoring them weekly for 3 weeks, fortnightly for 2 months, then monthly for 9 months, and quarterly thereafter.

The second study cohort was the Sinikithemba cohort comprising of 450 antiretroviral naïve, subtype C chronically infected black South Africans enrolled from McCord Hospital in KwaZulu-Natal, Durban, South Africa from August 2003 to 2008 and followed-up longitudinally. ${ }^{16,17}$ The estimated time of infection for these participants is unknown. Samples from 324 participants were available for the present study. Sociodemographic characteristics, plasma viral load, and $\mathrm{CD}^{+}$T-cell count measurements were obtained at baseline (Table 1 ). $\mathrm{CD}^{+} \mathrm{T}$-cell counts and viral loads were measured every 3 and 6 months, respectively, from enrollment. Viral loads were determined using the automated Cobas Amplicor HIV-1 Monitor test (version 1.5; Roche Diagnostics). CD4 ${ }^{+} \mathrm{T}$ cells were enumerated using the Multitest kit (CD4/CD3/CD8/CD45) on a FACSCalibur flow cytometer (Becton Dickinson). 
TABLE 1. Baseline Characteristics of the CAPRISA 002 Acute Infection Cohort and Sinikithemba Chronic Infection Cohort

\begin{tabular}{|c|c|c|c|c|c|c|c|c|c|c|}
\hline \multirow[b]{2}{*}{ Characteristics* } & \multicolumn{5}{|c|}{$P P I A$ SNP rs6850 in CAPRISA 002 Acute Infection } & \multicolumn{5}{|c|}{$\begin{array}{c}P P I A \text { SNP rs6850 in Sinikithemba Chronic Infection } \\
\text { Cohort }\end{array}$} \\
\hline & $\mathbf{A A}(\mathbf{n}=23)$ & $A G(n=18)$ & GG $(n=6)$ & MAF & H-W & $\mathbf{A A}(n=146)$ & $A G(n=124)$ & GG $(n=54)$ & MFA & $\mathbf{H}-\mathbf{W}$ \\
\hline Age, yrs, median (IQR) & $25(22-37)$ & $25(19-31)$ & $23(21-25)$ & 0.35 & 0.55 & $30(27-34)$ & $29(25-34)$ & $31(26-38)$ & 0.36 & 0.003 \\
\hline Sex, female $(\%)$ & $23(100)$ & $18(100)$ & $6(100)$ & & & $115(78.8)$ & $98(79.0)$ & $43(79.6)$ & & \\
\hline $\begin{array}{l}\text { CD4 cells } / \mu \mathrm{L}, \text { median } \\
\quad(\mathrm{IQR})\end{array}$ & $535(420-877)$ & 877 (671-973) & $574(518-695)$ & & & $327(209-459$ & $394(256-518)$ & $357(281-579)$ & & \\
\hline $\begin{array}{l}\text { Log viral loads, median } \\
\text { (IQR) }\end{array}$ & $4.6(3.8-5.3)$ & $4.9(4.5-5.8)$ & $5.0(4.5-5.6)$ & & & $4.8(4.0-5.3)$ & $4.8(4.0-5.4)$ & $4.6(3.7-5.2)$ & & \\
\hline
\end{tabular}

*Age of participants is at recruitment into the study, viral and CD4 measurements are at time of HIV diagnosis.

$\mathrm{n}$, number of individuals; MFA, minor allele frequency; H-W, Hardy-Weinberg; IQR, interquartile range.

\section{Peripheral Blood Mononuclear Cells Sample Processing}

Peripheral blood mononuclear cells (PBMCs) were isolated by Ficoll-Histopaque (Sigma) density gradient centrifugation from blood within 6 hours of phlebotomy and frozen in liquid nitrogen until use. Ethical approval was obtained from the Biomedical Research Ethics Committee of the University of KwaZulu-Natal. All participants provided written informed consent.

\section{Genotyping for Regulatory SNPs (rs6850 and rs8177830) in the PPIA}

SNPs (rs8177830 and rs6850) were first genotyped in the aforementioned available DNA samples from CAPRISA 002 cohort by restriction fragment length polymorphism assay as previously reported. ${ }^{10}$ Briefly, DNA samples were amplified by PCR using Taq DNA polymerase (Invitrogen, Carlsbad, CA) in the presence of $1.25 \mathrm{mM} \mathrm{MgCl}_{2}$ with the following primer set: forward primer, CypAex-1s: 5'-AAGTCGCAGACCCGATTG-3' and reverse primer, CypA-1a: 5'-ACTTTCTGGGCCCCATTC-3'. Subsequently, PCR products were subjected to a restriction digest with RsaI and HaeIII (New England Biolabs, Ipswich, MA) overnight at $37^{\circ} \mathrm{C}$ and analyzed on a $4 \%$ agarose gel, to detect polymorphisms.

\section{CypA mRNA Expression Analysis and In Vitro Replication Assay}

PBMCs were isolated from a total of $28 \mathrm{SN}$ and $28 \mathrm{SC}$ participants with known genotypes for SNP rs6850 from the CAPRISA 002 cohort. There were at least 2 study time points available for each of the participants in the primary HIV-1 infection phase and a total of 75 separate samples were analyzed for this group. Thirteen matched samples were included in this analysis.

\section{RNA Isolation and Analysis}

For all samples, RNA was extracted immediately after thawing and counting of PBMCs without in vitro stimulation. RNA was extracted from $2 \times 10^{6}$ PBMCs using the RNeasy Mini Kit (Qiagen, Hilden, Germany). The total RNA concentration was quantified and samples were used only if the $\mathrm{OD}_{260} / \mathrm{OD}_{280}$ ratio was 1.90 or greater. All RNA samples were DNAse treated. Approximately $1 \mu \mathrm{g}$ of total RNA from each sample was reversed transcribed using the iScript cDNA synthesis kit (Biorad Laboratories, Inc, Berkeley, CA).

\section{Real-Time PCR RNA Quantitation}

CypA mRNA levels were analyzed by SYBR green qPCR using the LightCycler 480 (Roche). Each PCR reaction consisted of $3 \mathrm{mmol} / \mu \mathrm{L} \mathrm{MgCl} 2$, the respective primers at 0.5 $\mathrm{pmol} / \mu \mathrm{L}, 1 \mu \mathrm{L}$ Fast Start SYBR Green I (Roche), $1 \mu \mathrm{g}$ cDNA and water to make the total reaction volume to $10 \mu \mathrm{L}$. CypA cDNA was detected using the following primer set, CypA-F: 5'-GTCAACCCCACCGTGTTCTTC-3' and CypA-R: 5'-TTTCTGCTGTCTTTGGGACCTTG-3'. Glyceraldehyde 3-phosphate dehydrogenase (GAPDH) determined to be the most suitable reference gene based on PCR efficiency was used to correct for differences in the cDNA input, GAPDH cDNA was detected using the following primer set: GAPDH-F: 5'-GTCAACCCCACCGTGTTCTTC-3' and GAPDH-R: 5'-TTTCTGCTGTCTTTGGGACCTTG-3'. SYBR green qPCR was performed using the following program on the LightCycler 480: (1) preincubation: $95^{\circ} \mathrm{C}$ for 5 minutes; (2) amplification: 45 cycles of $95^{\circ} \mathrm{C}$ for 15 seconds, $60^{\circ} \mathrm{C}$ for 15 seconds, and $72^{\circ} \mathrm{C}$ for 15 seconds; (3) melting curve: $95^{\circ} \mathrm{C}$ for 5 seconds, $65^{\circ} \mathrm{C}$ for 1 minute, and $97^{\circ} \mathrm{C}$ for 0 seconds with a temperature transition rate of $0.11^{\circ} \mathrm{C} / \mathrm{sec}$. To confirm amplification specificity, the PCR products were subjected to a melting curve analysis. Serial dilutions of cDNA from total RNA were performed for each target gene to create standard curves for quantitative analysis.

\section{HIV-1 In Vitro Replication Assay}

HIV-1 replication capacity was assessed in PBMCs isolated from 20 healthy HIV uninfected individuals with known genotypes for SNP rs6850. PBMCs were isolated as aforementioned. PBMCs $\left(2 \times 10^{6} / \mathrm{mL}\right)$ were stimulated for 48 hours in R10 buffer [Roswell Park Memorial Institute (RPMI)-1640 medium (Grand Island, NY) supplemented with 10\% fetal calf serum (Hyclone Inc., Logan), gentamicin (Gibco-Brl, Gaithersburg, MD) $(100 \mathrm{mg} / \mathrm{mL})]$ containing $5 \mathrm{mg} / \mathrm{mL}$ phytohemagglutinin (PHA) (Roche, Indianapolis, IN) and interleukin2 (IL-2) (Roche). Following stimulation with PHA/IL-2, cells were washed with R10 buffer and then infected with HIV IIIB (NIH AIDS Reagent Repository) at $0.1 \mathrm{MOI}$ by spinoculation (2 hours, $2500 \mathrm{RPMs}, 37^{\circ} \mathrm{C}$ ). Infection was performed in a 24-well plate. Virus was subsequently removed by washing the cells, 
followed by cell culture for 10 days. CypA mRNA expression levels were analyzed by real-time PCR in these cultured cells. Cell culture supernatants from day 2, 7, and 10 were harvested and analyzed by p24 antigen capture enzyme-linked immunosorbent assay (Vironostika, Biomérieux).

\section{Statistical Analysis}

The difference in allele frequency distribution between the SN and SC group was determined by Fisher exact test for SNPs rs8177830 and rs6850 to test the null hypothesis that allele frequencies were the same between individuals carrying the wild-type and mutant genotypes.

The association of SNP rs6850 with $\mathrm{CD}^{+}{ }^{+} \mathrm{T}$-cell counts and HIV-1 viral loads was determined using a generalized estimating equation model ${ }^{18}$ taking into account longitudinal measures for each participant. Viral loads were logtransformed and the square root of $\mathrm{CD}^{+} \mathrm{T}$-cell counts was used to normalize their measurements.

In the CAPRISA 002 cohort, Kaplan-Meier survival curves and proportional hazards models were used to assess the effect of SNP rs6850 on time to HIV-1 acquisition after enrollment and on $\mathrm{CD}^{+}$T-cell decline to less than 350 cells per microliter $(C D 4<350)$. A linear mixed model was used to estimate the rate of $\mathrm{CD}^{+}{ }^{+} \mathrm{T}$-cell decline in participants harboring the wild-type versus mutant genotypes from the Sinikithemba cohort.

CypA mRNA expression levels were compared between SN and SC groups using nonparametric statistical analysis, and Pearson correlation. Values were expressed as median values. Differences between the groups were evaluated using Dunn multiple comparison test or the MannWhitney $U$ test. The software used for the analysis was GraphPad prism version 5 and SAS version 9.3 (SAS Institute Inc., Cary). A result was considered significant if the $P$-value was $<0.05$.

\section{RESULTS}

In the present study, we investigated the frequency of rs8177830 located and SNPs rs6850 in the regulatory region of PPIA in a total of 539 participants from 2 black South African HIV-1 cohorts; $168 \mathrm{SN}$ and $47 \mathrm{SC}$ participants were from the CAPRISA 002 cohort and 324 participants from the Sinikithemba chronic HIV-1 infection cohort (Fig. 1). rs8177830 G was infrequent (minor allele frequency $=0.01$ ) in the CAPRISA 002 cohort and therefore SNP rs8177830 was excluded from any further analysis and genotyping in the Sinikithemba chronic HIV-1 infection cohort.

\section{SNP rs6850 has No Direct Association With Susceptibility to HIV-1 Acquisition}

The study design of the CAPRISA 002 cohort in which participants at high risk for HIV-1 infection were enrolled while HIV-1 negative and followed longitudinally for 2 years, allowed us to study the association of SNP rs6850 with
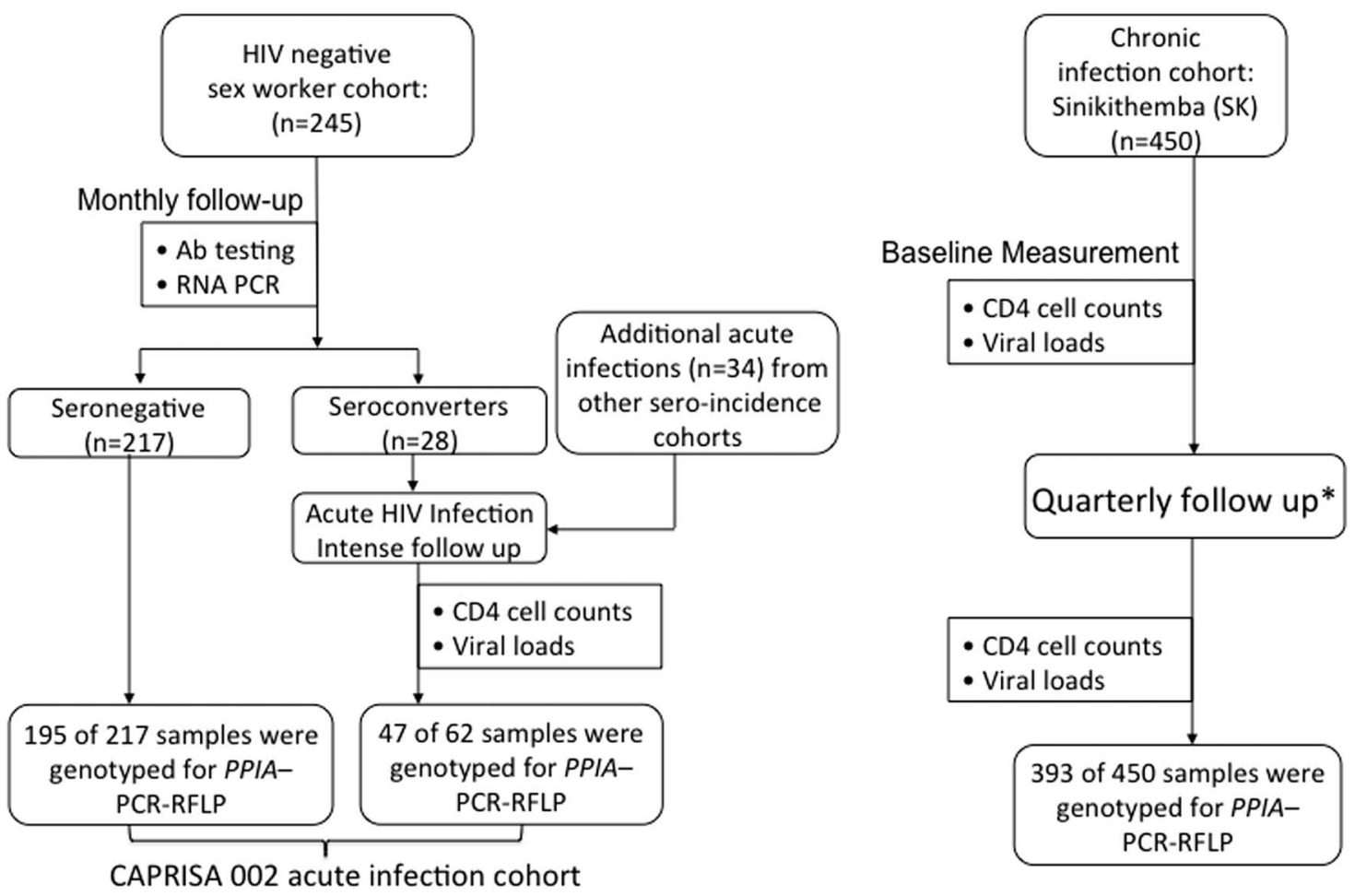

FIGURE 1. Design of South African HIV-1 cohorts used in this study and the number of participants genotyped from each group. *Quarterly follow-up is 3 monthly viral loads and 6 monthly CD4 cell counts measurements. 
TABLE 2. Effect of PPIA SNP rs6850 on HIV-1 Acquisition Risk

\begin{tabular}{|c|c|c|c|c|c|c|c|}
\hline \multirow[b]{3}{*}{ PPIA SNP } & \multirow[b]{3}{*}{ Risk Group } & \multirow[b]{3}{*}{$\mathbf{n}$} & \multicolumn{3}{|c|}{ Genotype } & \multicolumn{2}{|c|}{ SP Versus SN } \\
\hline & & & \multicolumn{3}{|c|}{ No. Carriers (Frequency) } & \multirow{2}{*}{$\begin{array}{c}\text { Hazard Ratio }(95 \% \text { CI) } \\
\text { AA Versus AG/GG } \\
\end{array}$} & \multirow[b]{2}{*}{$P$} \\
\hline & & & $\mathbf{A A}$ & AG & GG & & \\
\hline & $\mathrm{SC}$ & 47 & $23(0.49)$ & $18(0.38)$ & $6(0.13)$ & 1.57 (0.64 to 3.62$)$ & 0.31 \\
\hline
\end{tabular}

CI, confidence interval; n, number of individuals.

susceptibility to HIV-1 infection. There was no distortion in SNP rs6850 distribution between SN and SC participants (relative hazard, 1.57 ; $95 \% \mathrm{CI}: 0.64$ to $3.62, P=0.3067$ )
(Table 2). These data suggest that SNP rs6850 may not influence HIV-1 acquisition at least in a population of black South Africans.
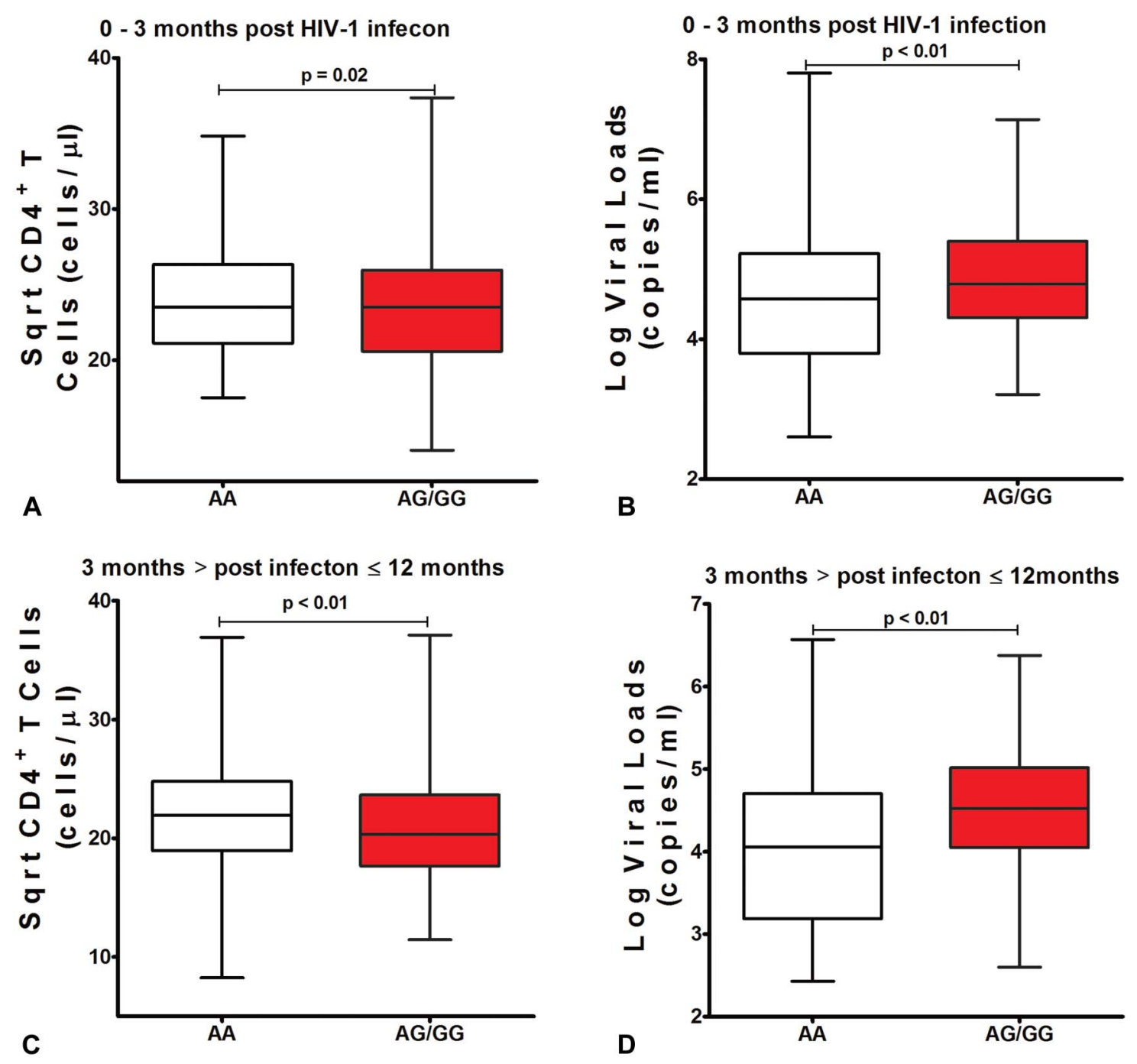

FIGURE 2. Association of SNP rs6850 with clinical outcomes during primary HIV-1 infection in the CAPRISA 002 cohort. The horizontal line in the middle of the whiskers plot denotes the median value. The differences between groups were evaluated using generalized estimating equation. $P<0.05$ denotes statistical significance. $A$ and $B$, association of rs $6850 \mathrm{G}$ with lower $C D 4^{+}{ }^{-}$-cell counts and higher viral loads, respectively, during the acute phase of infection. C and D, association of rs6850 G with lower CD4 ${ }^{+}$ T-cell counts and higher viral loads, respectively, during the early chronic phase of infection. 


\section{Influence of SNP rs6850 on CD4 ${ }^{+} \mathrm{T}$-cell Counts and Viral Loads}

A dominant model analysis using generalized estimating equation was used to compare $\mathrm{CD}^{+}$T-cell counts and viral loads between individuals who carried the wild-type genotype (rs6850AA) and those carrying mutant genotypes (rs6850AG/ GG) in the CAPRISA 002 cohort. Consistent with the ex vivo data reported by Bleiber et al, ${ }^{11}$ the $\mathrm{rs} 6850 \mathrm{G}$ was significantly associated with lower $\mathrm{CD}^{+}{ }^{+} \mathrm{T}$-cell counts (mean 506 versus 557 cells $/ \mu \mathrm{L}, P=0.026$ ) (Fig. $2 \mathrm{~A}$ ) and higher viral loads (mean 4.85 versus $4.46 \log$ copies $/ \mathrm{mL}, P<0.001$ ) (Fig. $2 \mathrm{~B}$ ) during the acute phase of infection ( $0-3$ months). Interestingly, rs 6850 $\mathrm{G}$ was also significantly associated with lower $\mathrm{CD}^{+}{ }^{+} \mathrm{T}$-cell counts (mean 442 versus 494 cells $/ \mu \mathrm{L}, P=0.001$ ) (Fig. $2 \mathrm{C}$ ) and higher viral loads (mean 4.49 versus $4.01 \log$ copies $/ \mathrm{mL}$, $P<0.001$ ) (Fig. 2D) during early chronic phase of infection (3-12 months). Given that the time of infection was known and specific time points were analyzed for black South Africans in the CAPRISA 002 cohort, our study suggests that rs6850 G may be associated with enhanced HIV-1 replication in vivo.

\section{Effect of SNP rs6850 on Disease Progression}

Next, we examined the effect of rs $6850 \mathrm{G}$ on disease progression as reflected by time to $\mathrm{CD} 4<350$ cells per microliter in SC from the CAPRISA 002 cohort. The rs6850 $\mathrm{G}$ was marginally associated with rapid $\mathrm{CD}^{+} \mathrm{T}$-cell decline (relative hazard, 3.18; 95\% CI: 1.01 to $10.04, P=0.048$ ) (Fig. 3A). This borderline association could be due to a small sample size (only $47 \mathrm{SC}$ from the CAPRISA 002 cohort).

Samples from 324 black South Africans in the Sinikithemba chronic HIV-1 infection cohort were available to study the association of SNP rs6850 with the rate of $\mathrm{CD}^{+}$T-cell decline during follow-up since enrolment. Interestingly, rs6850 $\mathrm{G}$ was significantly associated with rapid $\mathrm{CD}^{+} \mathrm{T}$-cell decline in the Sinikithemba chronic HIV-1 infection cohort during follow-up ( $P=0.016$ ) (Fig. 3B). Taken together, the data from the 2 black South African HIV-1 infection cohorts suggest that rs6850 G may be associated with rapid HIV-1 disease progression among black South Africans.

\section{Effect of rs6850 G on CypA Expression Levels In Vivo}

As rs6850 SNP is located in the upstream regulatory region of the PPIA gene, we reasoned that it may regulate the transcription levels of the gene. Therefore, we examined the association of rs6850 $\mathrm{G}$ with CypA mRNA expression levels in vivo. We performed quantitative reverse transcriptase PCR to measure CypA mRNA levels in PBMCs of $28 \mathrm{SN}$ and 28 SC participants. There were no differences in CypA mRNA expression levels between participants carrying rs6850AA and $\mathrm{rs} 6850 \mathrm{AG} / \mathrm{GG}$ in the SN group (Fig. 4A). These data suggest that CypA is not differentially expressed according to this allele in a general population. However, rs6850 G was significantly associated with elevated CypA mRNA expression levels following HIV-1 infection $(P=0.006)$ (Fig. 4B). The data from Figure 4B suggest a plausible mechanism where HIV-1 infection modulates CypA mRNA expression levels in the presence of regulatory region SNP, rs8650 G. Taken together, the expression data suggest that rs6850 G may not be associated with susceptibility to HIV-1 infection but with enhanced HIV-1 replication in vivo.

\section{Effect of rs6850 G on CypA Expression Levels and HIV-1 Replication Ex Vivo}

Last, we assessed HIV-1 replication capacity in PHA/ IL-2 stimulated PBMCs from 20 SN participants with known SNP rs6850 genotypes. CypA mRNA levels were measured in PHA/IL-2 stimulated PBMCs by performing quantitative

\section{CAPRISA AI 002 cohort}

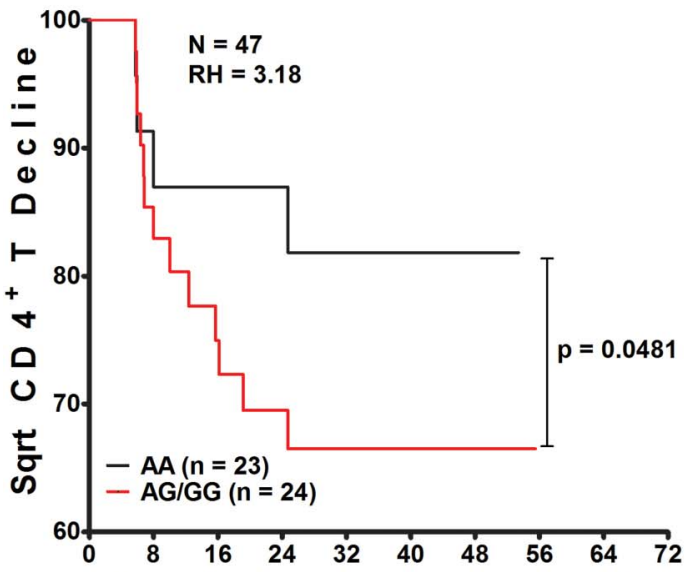

A Time since seroconversion, months
Sinikithemba Cohort

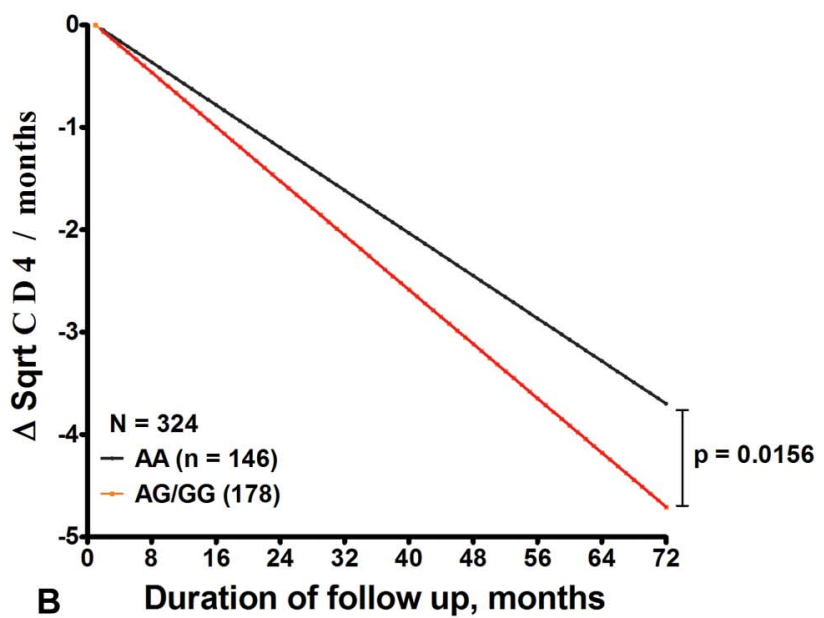

FIGURE 3. The association of SNP rs6850 with CD4+ T-cell decline. A, Kaplan-Meier survival curve analysis of SNP rs6850 for $\mathrm{CD}^{+} \mathrm{T}$ decline in the CAPRISA 002 cohort. B, Rates of CD4 ${ }^{+} \mathrm{T}$-cell decline stratified by genotypes for SNP rs6850. CD4 ${ }^{+} \mathrm{T}$-cell decline in participants harboring the wild-type genotype (rs6850AA) is shown by a black curve, whereas the red curve represents CD4+ T-cell decline in participants harboring mutant genotypes (rs6850AG/GG). 
Seronegative participants

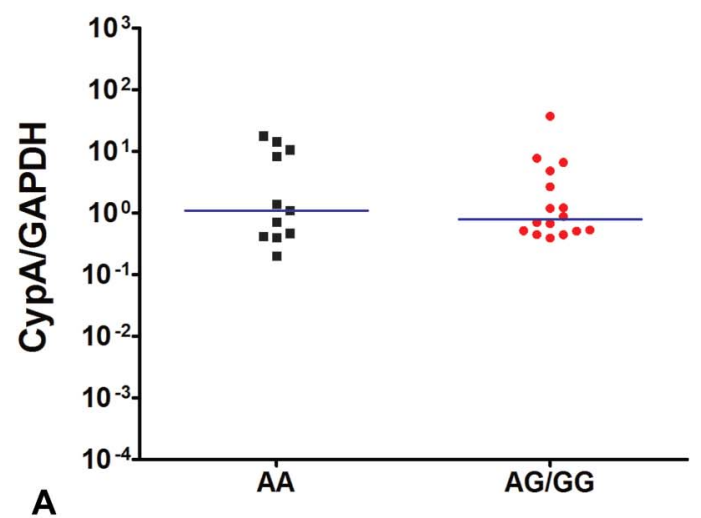

A

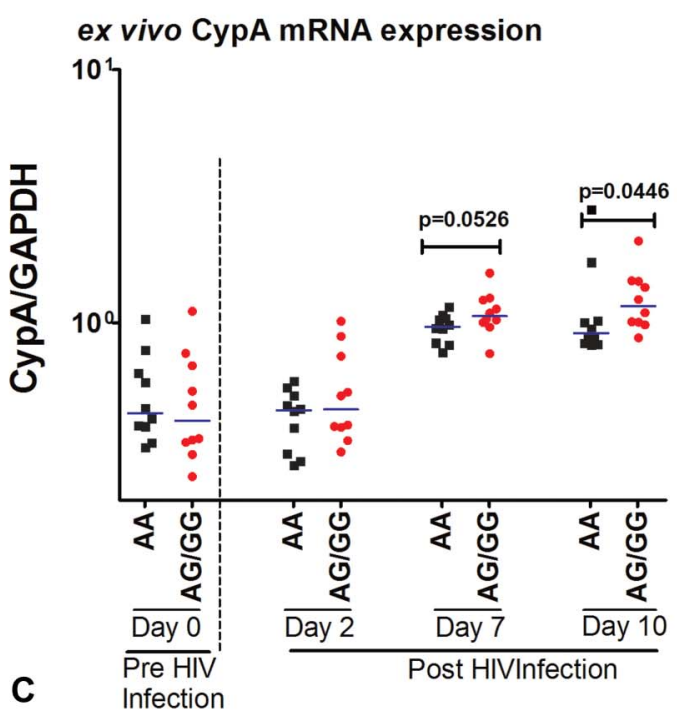

HIV-1 positive participants
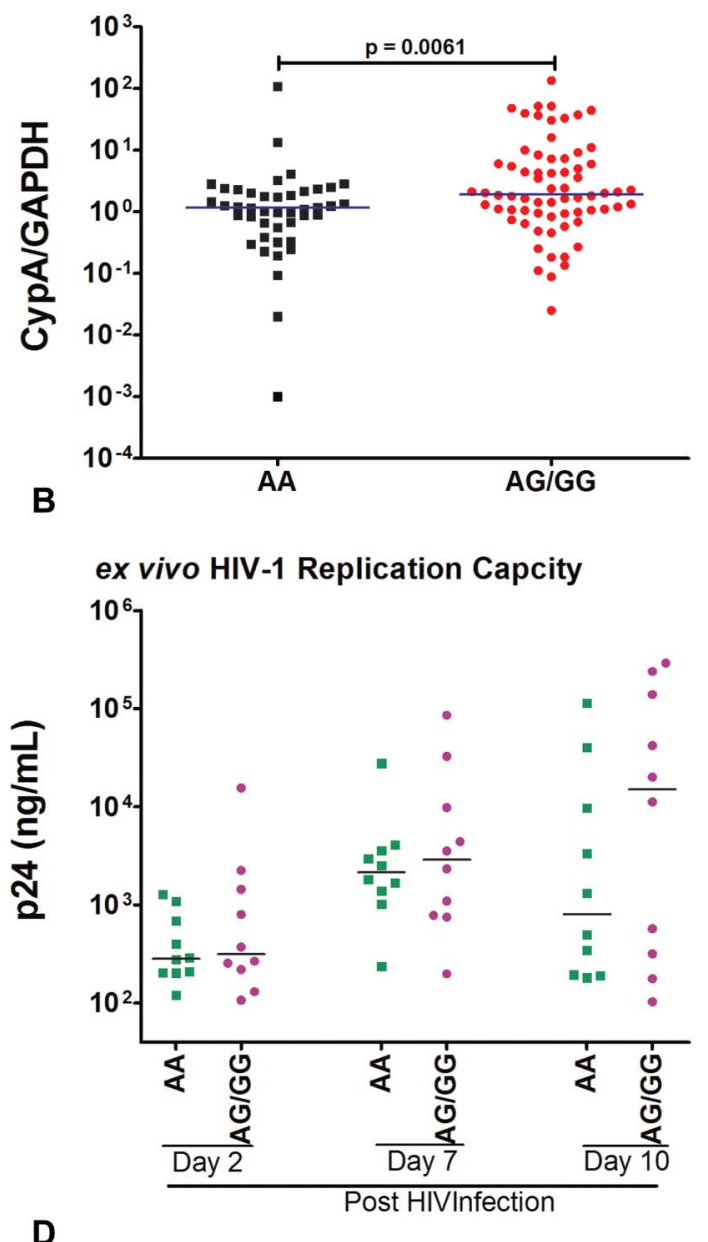

FIGURE 4. Expression of CypA mRNA levels in PBMCs obtained from participants in the CAPRISA 002 cohort. Data are represented as the normalized ratio of CypA to GAPDH. The horizontal line in the middle of the points denotes the median value. The differences between groups were evaluated using Mann-Whitney $U$ test. $P<0.05$ denotes statistical significance. A, CypA mRNA expression levels in PBMCs from nonseroconverters. B, CypA mRNA expression levels in PBMCs from primary infected participants. C, CypA mRNA expression levels in PHA/IL2 stimulated PBMCs obtained from seronegative participants with known genotypes and subsequently infected with HIV IIIB. D, HIV-1 replication capacity in PBMCs with known genotypes as measured by p24 production.

reverse transcriptase PCR before and after HIV-1 infection. There were no differences in CypA mRNA expression levels between PBMCs harboring 6850AA and 6850AG/GG before HIV-1 infection, whereas rs6850 G variant was associated with higher CypA mRNA expression levels at day 7 and day 10 postinfection $(P=0.053$ and $P=0.045$, respectively; Fig. $4 C)$. Although there was a borderline association of rs6850 with higher CypA mRNA expression after in vitro HIV-1 infection of PBMCs probably because of short infection period (up to 10 days), these data further suggest that CypA may be modulated by HIV-1 infection among individuals harboring rs6850 SNP. These data suggest that rs6850 may be associated with enhanced HIV-1 replication and/or disease progression but susceptibility to HIV-1 acquisition.

In an attempt to generate further evidence for the possible role of the rs6850 $\mathrm{G}$ polymorphism in promoting HIV-1 replication, we analyzed the replicative capacity of HIV-1 in PHA/IL-2 stimulated PBMCs by p24 antigen enzyme-linked immunosorbent assay measurements. There was nonsignificant trend of association of $\mathrm{rs} 6850 \mathrm{G}$ with higher p24 production levels (Fig. 4D). Lack of significant difference in p24 production could be attributed to a shorter inoculation period of PBMCs with the virus consistent with the data of the previous study. ${ }^{12}$ Taken together, these data suggest that rs6850 G promotes efficient HIV-1 replication through elevated PPIA expression levels in the context of HIV-1 infection.

\section{DISCUSSION}

The role of CypA in promoting HIV-1 replication was initially established through extensive in vitro experiments. ${ }^{2,3,7,8}$ Subsequent studies investigated the association 
of CypA genetic variation with HIV-1 disease outcome in different population groups, as one powerful approach to confirm the importance of CypA in vivo. ${ }^{10-12}$ rs6850 $\mathrm{G}$ in the regulatory region of $P P I A$ was associated with increased susceptibility to HIV-1 acquisition in European Americans ${ }^{10}$ while it was shown to be associated with protection against HIV-1 infection in the ACS. ${ }^{12}$ Given the discrepancies in the data obtained from previous studies, we wanted to elucidate the role of CypA genetic variation in HIV-1 acquisition and disease progression in an African setting with high HIV prevalence. Contrary to the previous studies, ${ }^{10,12}$ there was no evidence of association of $\mathrm{rs} 6850 \mathrm{G}$ with susceptibility to HIV-1 infection in our cohort. One possible reason for the discrepancies in results between studies is that allele frequencies vary among potential disease-influencing gene variants according to ethnic groups and geographically separated populations. ${ }^{19}$ We speculate that there may be other modifying biological factors that affect the interaction of this SNP with HIV-1 following exposure to the virus thus resulting in divergent data depending on cohort or study population analyzed. The presence of another SNP that may be in linkage disequilibrium with SNP rs6850 could not be excluded from the reported possible influence of rs6850 G on susceptibility to HIV-1 infection. Therefore, the role of rs6850 $\mathrm{G}$ in susceptibility to HIV-1 infection remains inconclusive and warrants further investigation. Although SNP rs8177826, a proxy for SNP rs8177830, was associated with rapid progression to AIDS in the American population ${ }^{10}$ and among European drug users, ${ }^{12}$ SNP rs8177830 was very rare in the South African study population and therefore could not be analyzed for association with HIV-1 infection outcome in the present study.

Our data demonstrate that rs6850 G was significantly associated with lower $\mathrm{CD}^{+}{ }^{+} \mathrm{T}$-cell counts and higher viral loads as early as 3 months post-HIV-1 infection, confirming the importance of CypA in promoting HIV-1 replication as was suggested by in vitro data. ${ }^{2,3,7,8}$ Interestingly, rs6850 was further associated with rapid $\mathrm{CD}^{+}{ }^{+} \mathrm{T}$-cell decline in both black South African cohorts. Although there was no clear association of SNP rs6850 with $\mathrm{CD}^{+}$T-cell decline in the African or European American populations, ${ }^{10}$ Bleiber et $\mathrm{al}^{11}$ and our data clearly demonstrate the association of rs6850 $\mathrm{G}$ with rapid $\mathrm{CD}^{+}$decline. Taken together, our data suggest an association of CypA genetic variation with HIV-1 disease outcome.

Consistent with the previous study, ${ }^{12}$ SNP rs6850 was not associated with CypA expression in a general population of SN. However, our results demonstrate an increase in CypA mRNA expression levels in PBMCs isolated from SC participants carrying rs6850AG/GG compared with PBMCs from rs6850AA carriers. These data suggest that rs6850 G enhances HIV-1 replication by upregulating the CypA expression levels following HIV-1 infection. Following ex vivo HIV-1 infection of PBMCs obtained from SN participants with known SNP rs6850 genotypes, CypA mRNA levels increased in PBMCs obtained from rs6850AG/GG compared with rs6850AA carriers. Consistent with the in vivo data, rs6850 $\mathrm{G}$ supported high ex vivo viral replication compared with rs6850A as was reflected by p24 production. In light of the Gel Shift assay from An et al, ${ }^{10}$ it can be postulated that rs6850, which is in the regulatory region of PPIA, enhances the interaction of HIV-1 capsid (CA) with CypA leading to the incorporation of more CypA into the HIV-1 virion capsid. Increased CypA mRNA levels in rs6850AG/GG PBMCs following ex vivo HIV-1 infection may suggest an unknown mechanism where HIV-1 infection dysregulate CypA mRNA expression. Taken together, our results suggests that rs6850 $\mathrm{G}$ is associated with differential gene expression in the context of HIV infection, enhanced HIV-1 replication, and rapid $\mathrm{CD}^{+}$T-cell decline among black South Africans.

The major limitation of the present study is the small sample size especially for the cohort with known time of infection. It would be informative to study these polymorphisms in a larger sub-Saharan African cohort with known time of HIV-1 infection. It is worth pointing out that SNP rs6850 was not detected as having a significant effect on viral set point in genome-wide association studies, ${ }^{20,21}$ which could imply a more modest effect on HIV-1 clinical outcomes compared with the HLA locus or possibly because of population/ethnic differences in allele frequencies because the genome-wide association studies were performed in nonAfrican populations, with the exception of one study that found no association between genetic polymorphisms and HIV-1 susceptibility. ${ }^{22}$ Other reasons could be cohort design and outcome measures used, Pereyra et al investigated genetic variants associated with elite HIV-1 control $^{20}$ and Fellay et $\mathrm{al}^{21}$ studied genetic association with viral load set point.

In conclusion, our study demonstrates that the rs6850 G variant in the PPIA gene is associated with enhanced HIV-1 replication in 2 black South African HIV-1 cohorts. These findings are consistent with a crucial role for CypA in productive HIV-1 infection and lend support to ongoing efforts to develop antiretroviral drugs that can block the interaction between HIV-1 proteins and cellular proteins to disrupt the virus life cycle.

\section{ACKNOWLEDGMENTS}

The authors thank Yuchun Zhou and Beth BinnsRoemer for excellent technical support. The authors acknowledge the participants and their clinicians who participated in the CAPRISA 002 and HPP Sinikithemba cohorts.

\section{REFERENCES}

1. Braaten D, Franke EK, Luban J. Cyclophilin A is required for the replication of group $M$ human immunodeficiency virus type 1 (HIV-1) and simian immunodeficiency virus SIV(CPZ) GAB but not group $\mathrm{O}$ HIV-1 or other primate immunodeficiency viruses. J Virol. 1996;70: 4220-4227.

2. Luban J, Bossolt KL, Franke EK, et al. Human immunodeficiency virus type 1 Gag protein binds to cyclophilins A and B. Cell. 1993;73: 1067-1078.

3. Franke EK, Yuan HE, Luban J. Specific incorporation of cyclophilin A into HIV-1 virions. Nature. 1994;372:359-362.

4. Bosco DA, Eisenmesser EZ, Pochapsky S, et al. Catalysis of cis/trans isomerization in native HIV-1 capsid by human cyclophilin A. Proc Natl Acad Sci U S A. 2002;99:5247-5252.

5. Gamble TR, Vajdos FF, Yoo S, et al. Crystal structure of human cyclophilin A bound to the amino-terminal domain of HIV-1 capsid. Cell. 1996;87:1285-1294. 
6. Braaten D, Ansari H, Luban J. The hydrophobic pocket of cyclophilin is the binding site for the human immunodeficiency virus type $1 \mathrm{Gag}$ polyprotein. J Virol. 1997;71:2107-2113.

7. Thali M, Bukovsky A, Kondo E, et al. Functional association of cyclophilin A with HIV-1 virions. Nature. 1994;372:363-365.

8. Braaten D, Luban J. Cyclophilin A regulates HIV-1 infectivity, as demonstrated by gene targeting in human T cells. EMBO J. 2001;20: 1300-1309.

9. Sokolskaja E, Luban J. Cyclophilin, TRIM5, and innate immunity to HIV-1. Curr Opin Microbiol. 2006;9:404-408.

10. An P, Wang LH, Hutcheson-Dilks H, et al. Regulatory polymorphisms in the cyclophilin A gene, PPIA, accelerate progression to AIDS. Plos Pathog. 2007;3:e88.

11. Bleiber G, May M, Martinez R, et al. Use of a combined ex vivo/in vivo population approach for screening of human genes involved in the human immunodeficiency virus type 1 life cycle for variants influencing disease progression. J Virol. 2005;79:12674-12680.

12. Rits MA, van Dort KA, Kootstra NA. Polymorphisms in the regulatory region of the Cyclophilin A gene influence the susceptibility for HIV-1 infection. PLoS One. 2008;3:e3975.

13. UNAIDS. UNAIDS Report on the Global AIDS Epidemic. Geneva, Switzerland. World Health Organization, 2012.

14. Brockman MA, Schneidewind A, Lahaie M, et al. Escape and compensation from early HLA-B57-mediated cytotoxic T-lymphocyte pressure on human immunodeficiency virus type $1 \mathrm{Gag}$ alter capsid interactions with cyclophilin A. J Virol. 2007;81:12608-12618.

15. van Loggerenberg F, Mlisana K, Williamson C, et al. Establishing a cohort at high risk of HIV infection in South Africa: challenges and experiences of the CAPRISA 002 acute infection study. PLoS One. 2008;3:e1954.

16. Kiepiela P, Ngumbela K, Thobakgale C, et al. CD8+ T-cell responses to different HIV proteins have discordant associations with viral load. Nat Med. 2007;13:46-53.

17. Madlala P, Gijsbers R, Christ F, et al. Association of polymorphisms in the LEDGF/p75 gene (PSIP1) with susceptibility to HIV-1 infection and disease progression. AIDS. 2011;25:1711-1719.

18. Zeger SL, Liang KY. Longitudinal data analysis for discrete and continuous outcomes. Biometrics. 1986;42:121-130.

19. Winkler C, An P, O'Brien SJ. Patterns of ethnic diversity among the genes that influence AIDS. Hum Mol Genet. 2004;13:R9-R19.

20. Pereyra F, Jia X, McLaren PJ, et al. The major genetic determinants of HIV-1 control affect HLA class I peptide presentation. Science. 2010; 330:1551-1557.

21. Fellay J, Shianna KV, Ge D, et al. A whole-genome association study of major determinants for host control of HIV-1. Science. 2007;317: 944-947.

22. Petrovski S, Fellay J, Shianna KV, et al. Common human genetic variants and HIV-1 susceptibility: a genome-wide survey in a homogeneous African population. AIDS. 2011;25:513-518. 